\title{
Electric Field Measurements Reveal the Pivotal Role of Cofactor- Substrate Interaction in Dihydrofolate Reductase Catalysis
}

\author{
Aduragbemi S. Adesina, ${ }^{\S}$ Katarzyna Świderek, ${ }^{\S}$ Louis Y. P. Luk, Vicent Moliner,*
} and Rudolf K. Allemann*

Cite This: ACS Catal. 2020, 10, 7907-7914

Read Online

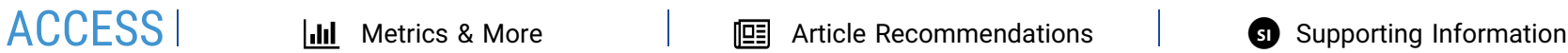

ABSTRACT: The contribution of ligand-ligand electrostatic interaction to transition state formation during enzyme catalysis has remained unexplored, even though electrostatic forces are known to play a major role in protein functions and have been investigated by the vibrational Stark effect (VSE). To monitor electrostatic changes along important steps during catalysis, we used a nitrile probe (T46C-CN) inserted proximal to the reaction center of three dihydrofolate reductases (DHFRs) with different biophysical properties, Escherichia coli DHFR (EcDHFR), its conformationally impaired variant (EcDHFR-S148P), and Geobacillus stearothermophilus DHFR (BsDHFR). Our combined experimental and computational approach revealed that the electric Ligand interaction controls electrostatics

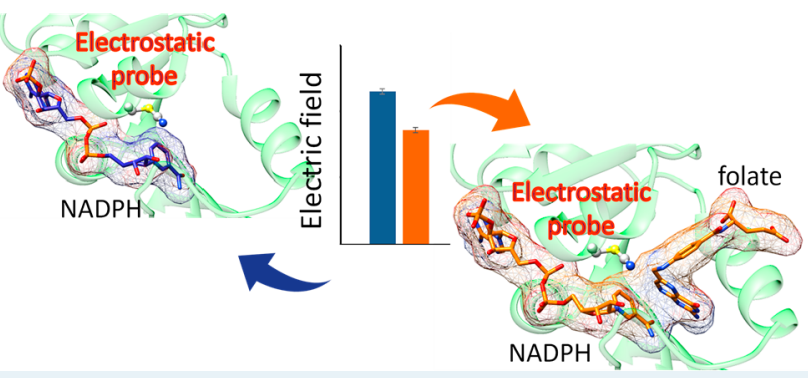
field projected by the substrate toward the probe negates those exerted by the cofactor when both are bound within the enzymes. This indicates that compared to previous models that focus exclusively on subdomain reorganization and protein-ligand contacts, ligand-ligand interactions are the key driving force to generate electrostatic environments conducive for catalysis.

KEYWORDS: dihydrofolate reductase, vibrational Stark effect, protein FTIR, protein dynamics, protein electrostatic, ligand-ligand interaction

\section{INTRODUCTION}

Electrostatic effects are critical for protein function. ${ }^{1,2}$ In a typical enzymatic reaction, association and dissociation of substrates, cofactors, and products lead to fluctuations in the enzyme electrostatic potential. ${ }^{3}$ In some cases, such changes can induce structural reorganization, altering subdomain contacts and protein-ligand interactions. ${ }^{4,5}$ In addition, this reorganization can change ligand-ligand electrostatic interactions vital for catalysis. ${ }^{6,7}$ Previous studies have characterized the role of subdomain reorganization and protein-ligand interaction in enzyme-catalyzed reactions. During enzyme catalysis, reactants must be brought in close proximity in order to reach the effective concentration necessary for the reaction. Nevertheless, the contribution of ligand-ligand interactions in enzyme catalysis has not been investigated.

DHFR has long served as a paradigm to investigate the biophysical basis of enzyme catalysis. ${ }^{8,9}$ This enzyme catalyzes the transfer of a hydride from the $\mathrm{C}_{4}$ of $\mathrm{NADPH}$ to the $\mathrm{C}_{6}$ of protonated 7,8-dihydrofolate (DHF) to produce 5,6,7,8tetrahydrofolate (THF) (Figure 1A). The DHFR from Escherichia coli (EcDHFR) has been central to many investigations due to the large-scale conformational changes that are associated with the catalytic cycle. ${ }^{10}$ EcDHFR adopts two major conformations along its catalytic cycle, switching from the closed conformation in the reactant complex to the occluded form in the product complex (Figure $1 \mathrm{~B}$ and $1 \mathrm{C}) .^{10}$ In between these two states, the M20 loop (residues 9-23) interacts with the FG loop (residues 116-132) in the closed conformer and GH loop (residues 142-149) in the occluded conformer (Figure 1C). ${ }^{10}$ Several techniques have been employed to investigate the role of these loop movements for EcDHFR catalysis, including the use of orthogonal infrared probes to monitor electrostatic changes within the enzyme's active site. ${ }^{4,11-13}$ According to these studies, electrostatic changes in EcDHFR correlate strongly with its conformational dynamics, which has also been implicated in forming the transition state during EcDHFR catalysis.,11-13 However, crystal structures and NMR relaxation studies on DHFRs isolated from other organisms did not reveal such conformational flexibility; instead, the M20 loops remain in the closed conformation throughout the catalytic cycle. ${ }^{9,14}$ Other structural analyses have also suggested that specific loop-

Received: April 26, 2020

Revised: June 18, 2020

Published: June 19, 2020 


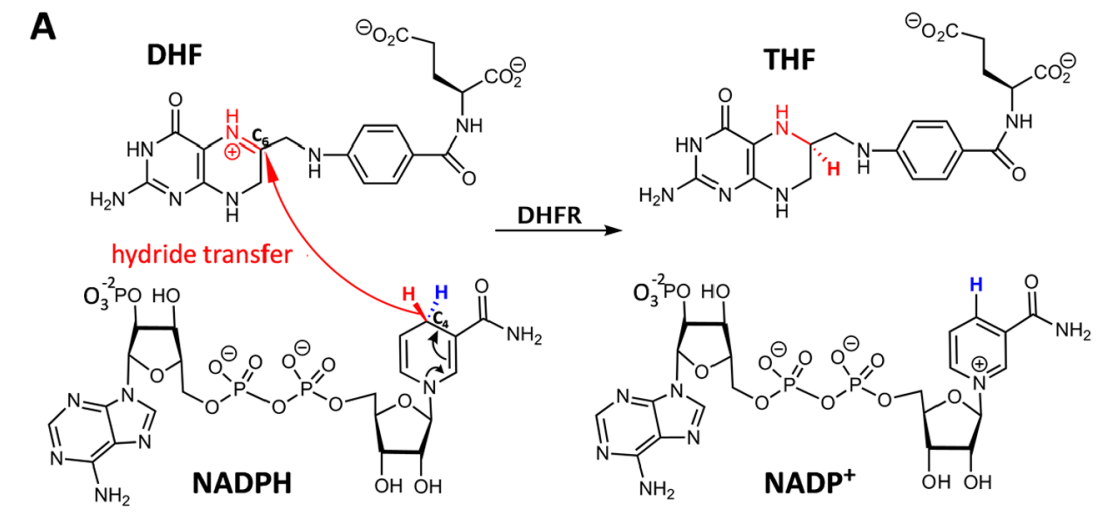

B
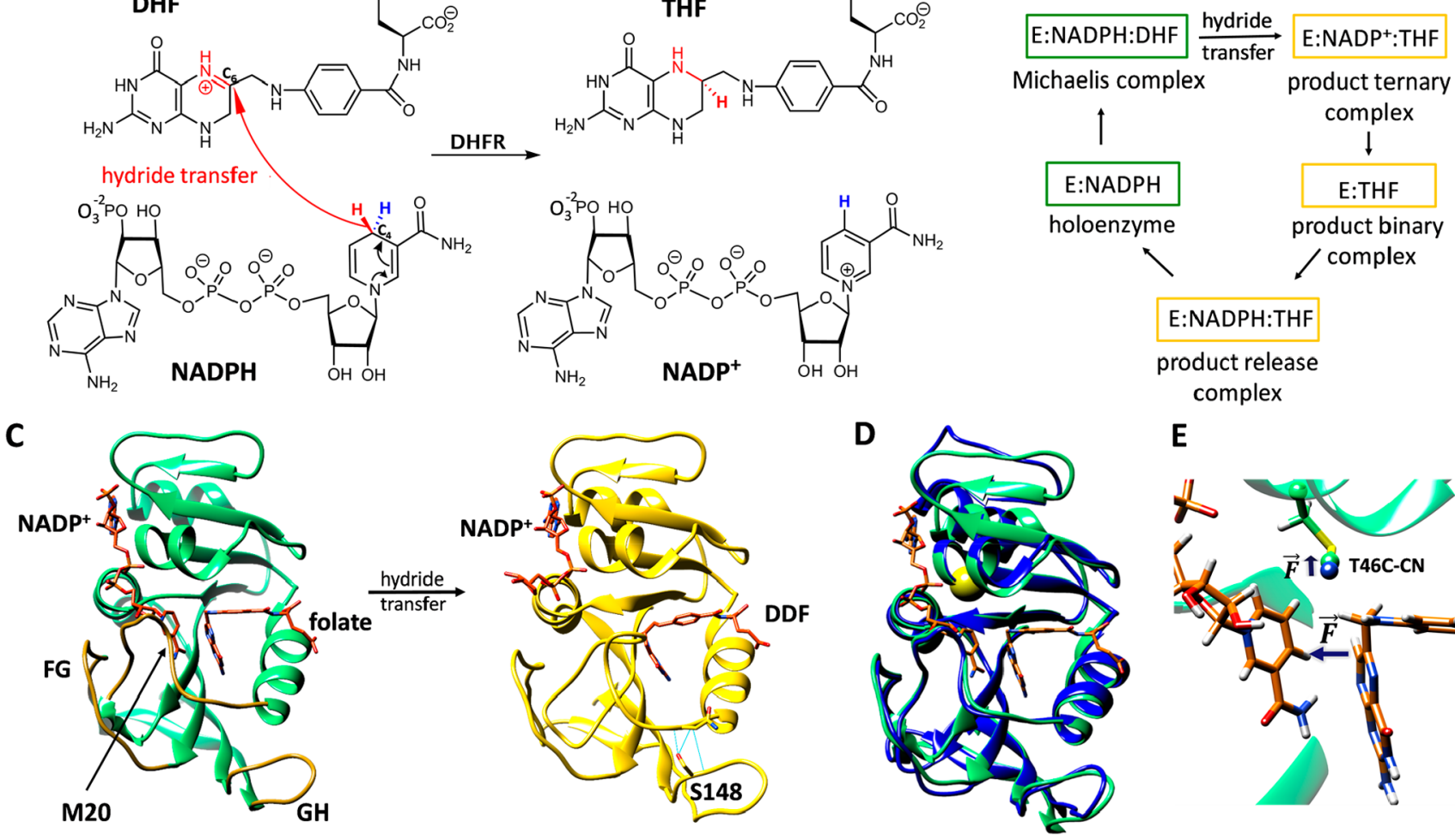

E

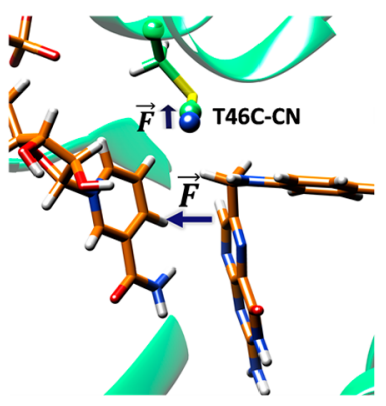

Figure 1. (A) Reaction catalyzed by dihydrofolate reductase (DHFR). (B) Catalytic cycle of EcDHFR showing the five catalytic steps and the two major enzyme conformations adopted; green boxes denote the closed conformation and gold the occluded conformation. (C) Cartoon representation of EcDHFR showing the catalytic loops and conformation transition between the closed (green, PDB 1RX2) and the occluded conformation (gold, PDB 1RX6; DDF, dideazatetrahydrofolate is a mimic of the product, THF). ${ }^{10}$ A variant of EcDHFR was generated where Ser148 was mutated to Pro to disrupt hydrogen bonding between M20 and GH loop, hindering formation of the occluded conformation. ${ }^{10}$ (D) Overlay of the cartoon representations of EcDHFR (green, PDB 1RX2), ${ }^{10}$ and BsDHFR (blue, PDB 1ZDR) ${ }^{20}$ in their reactive closed conformations. Position of the infrared probe (Thr46) is shown as a yellow sphere. (E) Close-up view of the active site of EcDHFR in the Michaelis complex after $5 \mathrm{~ns}$ simulation shows the absolute direction of the probe (T46C-CN) and resultant local electric fields.

ligand interaction in DHFR could facilitate catalysis, ${ }^{15,16}$ but this is not widely observed in all homologues. ${ }^{17}$

While earlier investigations of protein electrostatics relied exclusively on computational analysis, ${ }^{1,2}$ VSE spectroscopy has been developed into an experimental technique to elucidate the relationship between electrostatic potential and protein function. ${ }^{18,19}$ VSE originates from the intrinsic response of an infrared probe to an externally applied electric field. This calibration is then employed to calculate the magnitude of the electrostatic force experienced by the probe within a protein. ${ }^{18}$ Here, using VSE and multiscale quantum mechanics/molecular mechanics (QM/MM) simulations, we probe electrostatic changes within the active site of three DHFRs with diverse conformational flexibilities. A nitrile probe was inserted within $\sim 5 \AA$ of the reaction center of the enzymes by mutating an active site residue (Thr46) to a cysteine and subsequently labeling the sulfhydryl group with a nitrile. ${ }^{21}$ The response of the transition dipole of the probe in the flexible EcDHFR was compared to the significantly less flexible DHFR from Geobacillus stearothermophilus and the S148P variant of EcDHFR (EcDHFR-S148P) using different combinations of ligand that mimic important steps along their catalytic cycle. This approach revealed that the cofactor induces a strong electric field when bound within the active site, but this electric field becomes attenuated in the Michaelis complex where both the substrate and the cofactor are bound. Investigation of this attenuation using QM/MM simulations on EcDHFR reveals that electrostatic changes in the enzymes result from counteracting electric fields projected by the cofactor and substrate when they are bound within the active site

\section{RESULTS AND DISCUSSION}

2.1. Vibrational Stark Effect Measurements. Crystallographic studies of EcDHFR labeled with nitrile at position 46 revealed both the distance and the absolute direction of the probe within the active site (Figure 1E). ${ }^{11}$ Positioned about 5 $\AA$ from the reaction center, the probe allowed electric field changes projected between the ligands to be measured. Since labeling with nitrile did not significantly perturb the enzyme's properties (Figures S1 and S2 and Tables S1 and S2), ${ }^{11}$ the absorption frequencies of the probe in the apoenzyme, holoenzyme (E:NADPH), E:NADP ${ }^{+}$:folate, which mimics the Michaelis complex, and the product ternary complex $\left(\mathrm{E}: \mathrm{NADP}^{+}: \mathrm{THF}\right)$ were recorded using a high-resolution FTIR spectrophotometer equipped with a nitrogen-cooled MCT detector. The difference in absorption frequencies between the complexes was subsequently converted to local electrostatic changes using eq 1

$$
h c \Delta \bar{v}_{\text {obs }}=-\Delta \vec{\mu}_{\text {probe }} \cdot \Delta \vec{F}_{\text {protein }}
$$

where $h$ is Planck's constant, $c$ is the speed of light, $\Delta \vec{\mu}_{\text {probe }}$ represents the difference in the dipole moments of the ground 
and the excited states expressed as the linear Stark tuning rate $\left(\mathrm{cm}^{-1} /(\mathrm{MV} / \mathrm{cm})\right), \Delta \vec{F}_{\text {protein }}$ is the local electric field change experienced by the probe in the protein (in $\mathrm{MV} / \mathrm{cm}$ ) and $\Delta \tilde{v}_{\text {obs }}$ is vibrational frequency shift $\left(\mathrm{cm}^{-1}\right) .{ }^{21}$

Stark tuning rates for nitrile probes $\mid \Delta \vec{\mu}_{C N}$ If were determined and given as $\sim 0.7 \mathrm{~cm}^{-1} /(\mathrm{MV} / \mathrm{cm}){ }^{22}$ The local correction factor $f$ is between 1 and 1.8. ${ }^{23,24}$ However, for simplicity and easy comparison with existing publications, ${ }^{11,23} f$ is taken as unity here. Direct hydrogen bonding to the probe can lead to deviations of the observed vibrational frequency from the Stark model. ${ }^{25}$ Hence, NMR spectra of ${ }^{13} \mathrm{C}$-nitrilelabeled enzymes were measured and compared to a tandem FTIR-NMR calibration curve developed to correct for such deviations. ${ }^{25}$ Analysis with ${ }^{13} \mathrm{C}$-nitrile-labeled enzymes bound in the same complexes as in the VSE measurements revealed a common deviation of $\sim 7 \mathrm{~cm}^{-1}$ (Figure S11) similar to a previous report; ${ }^{11}$ thus, hydrogen-bonding correction was unneccesary. The enzymes $(2-5 \mathrm{mM})$ were mixed with a 10 fold excess of each ligand, and the transition dipole of the probe was recorded. When EcDHFR was mixed with $\operatorname{NADP}(\mathrm{H})$ to form the holoenzyme, a high-frequency absorption of $2166 \pm 0.2 \mathrm{~cm}^{-1}$ was observed (Figure 2 and

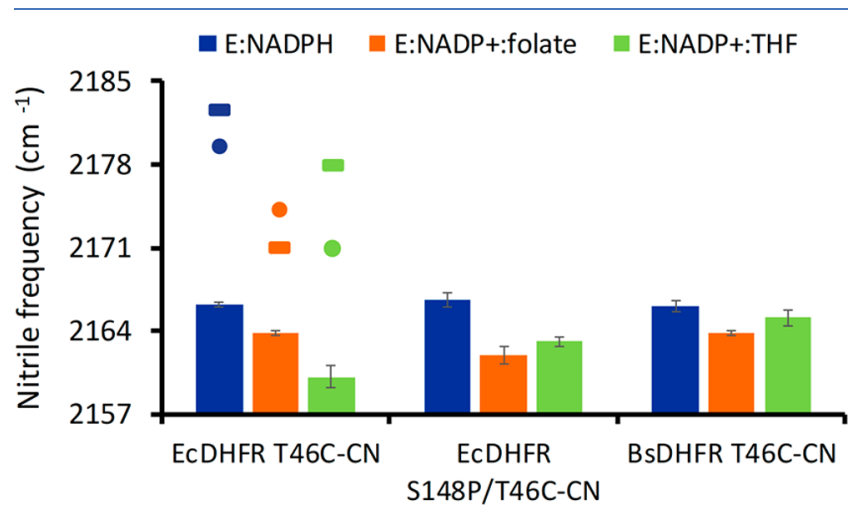

Figure 2. Bar charts showing the vibrational frequencies of the probe in the holoenzymes (E:NADPH, blue), the Michaelis complexes $\left(\mathrm{E}: \mathrm{NADP}^{+}\right.$:folate, orange), and the product ternary complexes (E:NADP ${ }^{+}$:THF, green) of EcDHFR, EcDHFR-S148P, and BsDHFR. Average vibrational frequencies from computational calculations of EcDHFR when randomly selected structures from the 501 simulated structures were used are represented as rectangles, while circles represent when only the last 50 ps structures are used. Error bars are omitted for clarity.

Table S3). However, this high-energy absorption decreases significantly when folate was added to the holoenzyme complex $\left(2164 \pm 0.2 \mathrm{~cm}^{-1}\right)$, and a further decrease was noted when the product (THF) was used to replace folate $\left(2160 \pm 0.9 \mathrm{~cm}^{-1}\right)$. The abortive E:NADP ${ }^{+}$:folate complex is an excellent mimic of the Michaelis complex (E:NADPH:DHF). ${ }^{26}$ The transition from the Michaelis complex $\left(\mathrm{E}: \mathrm{NADP}^{+}\right.$:folate $)$to the product ternary complex $\left(\mathrm{E}: \mathrm{NADP}^{+}\right.$:THF) resulted in the largest Stark shift, which agrees with previous studies that conformational change between the closed and the occluded forms induces electrostatic effects within the active site. ${ }^{4,11-13}$ Another noticeable Stark shift in EcDHFR is observed for the transition from the holoenzyme to the Michaelis complex.

When a similar analysis was carried out with the thermophilic BsDHFR, the probe also reported a high-energy absorption when the cofactor binds, giving a vibrational frequency of $2166 \pm 0.4 \mathrm{~cm}^{-1}$ (Figure 2), similar to that measured for EcDHFR. Similarly, the binding of folate to the holoenzyme of BsDHFR also caused a significant decrease in the high-energy absorption. However, the product ternary complex of BsDHFR differs from that of EcDHFR, so that transition between the Michaelis complex and the product ternary complex of BsDHFR resulted in a blue shift of $1.2 \mathrm{~cm}^{-1}$ corresponding to a local electric field change of $1.7 \mathrm{MV} / \mathrm{cm}$, whereas the same transition in EcDHFR showed a red shift of $-3.7 \mathrm{~cm}^{-1}(-5.3 \mathrm{MV} / \mathrm{cm})$.

Due to the difference observed for the product ternary complexes of EcDHFR and BsDHFR, the variant of EcDHFR (EcDHFR-S148P) that remains in the closed conformation throughout its catalytic cycle was tested. ${ }^{27}$ When electrostatic trends in the variant were measured, the results revealed that the S148P mutation altered the electric field, showing a similar trend as that of BsDHFR (Figure 2). Notably, the transition from the Michaelis complex to the product ternary complex in the variant resulted in a blue shift of $\sim 1.2 \mathrm{~cm}^{-1}$ (i.e., $1.7 \mathrm{MV}$ / $\mathrm{cm}$ ), which is identical to the value obtained for BsDHFR, suggesting that the significant electrostatic change between the Michaelis complex and the product ternary complex of EcDHFR is likely due to its conformational dynamics. The three enzymes showed similar high-energy absorptions in their holoenzyme complexes, independent of their temperature adaptation and flexibilities. In addition, they exhibited large electrostatic changes between the holoenzyme (E:NADP $(\mathrm{H})$ ) and the Michaelis complex $\left(\mathrm{E}: \mathrm{NADP}^{+}\right.$:folate) even though they adopt the closed conformation in both complexes, an observation also reported in a previous study when NADPH replaced $\mathrm{NADP}^{+}$in the Michaelis complex. ${ }^{11}$

2.2. $Q M / M M$ Simulations. To explore the origin of the high-frequency absorption in the holoenzyme and the electrostatic change that occurs in the Michaelis complex, $\mathrm{QM} / \mathrm{MM}$ simulations of EcDHFR in the different complexes were performed. First, we carried out an exhaustive benchmark analysis of different QM Hamiltonians (3 semiempirical methods and 18 density functionals) with a reduced gasphase model using MeCN and MeSCN. The BVP86 functional with a standard $6-311++g(d, p)$ basis set was found to reproduce the experimental frequencies of the molecules (Figure S12). Inclusion of anharmonicity has a negligible influence on the overall frequencies (Table S4). Calibration of the absorption frequency of the probe with $\mathrm{MeCN}$ and MeSCN shows a correlation between the Stark shifts and the charge redistribution when the electric fields are applied longitudinally to the nitrile bond (Figure S14)

Hybrid QM/MM simulations were then carried out on the three complexes of EcDHFR, including the closed holoenzyme (E:NADPH), the closed Michaelis complex (E:NADP ${ }^{+}$:folate), and the occluded product ternary complex (E:NADP $\left.{ }^{+}: T H F\right)$. Five hundred one random structures from each of the MD simulations of the complexes were selected for QM/MM calculations (Figures S16-S19). The QM/MM frequency distribution yielded average values of $2183.5 \pm 31.3$, $2169.9 \pm$ 8.5 , and $2177.8 \pm 14.0 \mathrm{~cm}^{-1}$ for the holoenzyme, Michaelis complex, and product ternary complex, respectively (Figures 2, rectangles, and 3). Indeed, our analysis agrees with the experiment, revealing that the binding of the cofactor induces a strong frequency absorption on the probe, which decreases significantly when the substrate binds. However, the simulations did not reproduce the trend observed in the product ternary complex. Indeed, the experimental trend was 


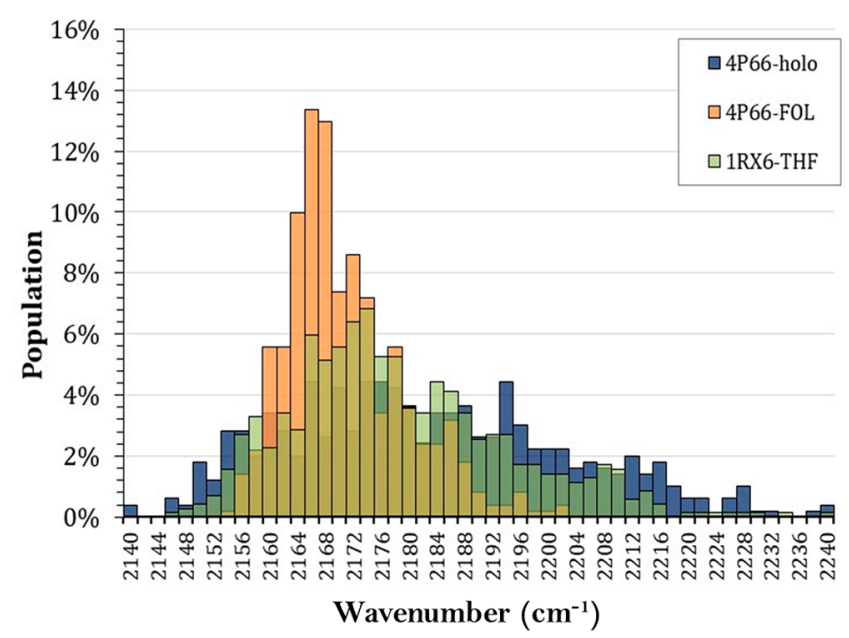

Figure 3. Distribution of frequencies of the nitrile bond computed at the BVP86/6-311++g(d,p)/MM level of theory for 501 structures of EcDHFR selected from MD simulations for the holoenzyme, $\mathrm{E}: \mathrm{NADPH}$ (blue), the Michaelis complex, E:NADP ${ }^{+}$:folate (orange), and the product ternary complex, E:NADP ${ }^{+}$THF (green).

reproduced entirely when only the last 50 ps simulations were used to compute the average frequencies, giving respective values of $2180.0 \pm 21.1,2173.6 \pm 6.6$, and $2169.8 \pm 13.5 \mathrm{~cm}^{-1}$ (Figure 2, circles, and Figure S19).

Although the system can be considered equilibrated based on the RMSD computed on the atoms of the backbone of the protein (Figure S16), the movement of the amino acid side chains during MD simulation can have a significant effect on the frequency of the probe, which is sensitive to changes within the microenvironment. This computational analysis challenged the previous analysis where the VSE of EcDHFR was quantitatively illustrated using computational studies. ${ }^{11} \mathrm{We}$ attributed this to the fact that the use of a few structures could provide the fortuitous agreement, as acknowledged by Hammes-Schiffer, Benkovic, and co-workers. ${ }^{11}$ Notably, the discrepancies between the trends relate to the inherent flexibility of EcDHFR, which limits the computational approach to accurately predict the vibrational frequency. Nevertheless, it shows that hydrogen-bonding rearrangement within the active site plays a crucial role in electrostatic preorganization (see Figure 4).

Further analysis reveals that the inherent flexibility of EcDHFR involves significant rearrangement of hydrogen bonding, leading to structures with similar electric fields but different vibrational frequencies and vice versa (Figure 4). This highlights the highly flexible nature of EcDHFR, which is different from enzymes previously investigated by IR spectroscopy. $^{24,28}$ The result, therefore, reinforces the need for large conformational sampling and rigorous statistical analysis of flexible proteins to accurately predict their Stark shifts. It is important to point out that the standard deviations reported for in silico calculations of single molecules originate from the statistical distribution and are distinct from experimental errors, which are obtained from ensemble averages. ${ }^{29}$

Continuing the computational analysis, the total electric field experienced by the probe in the three states (holoenzyme, Michaelis, and product complexes) were deconvoluted. Our simulations show that the cofactor propagates a high electric field in all of the complexes; it is the major contributor to the total electric field projected toward the probe (Table 1). In

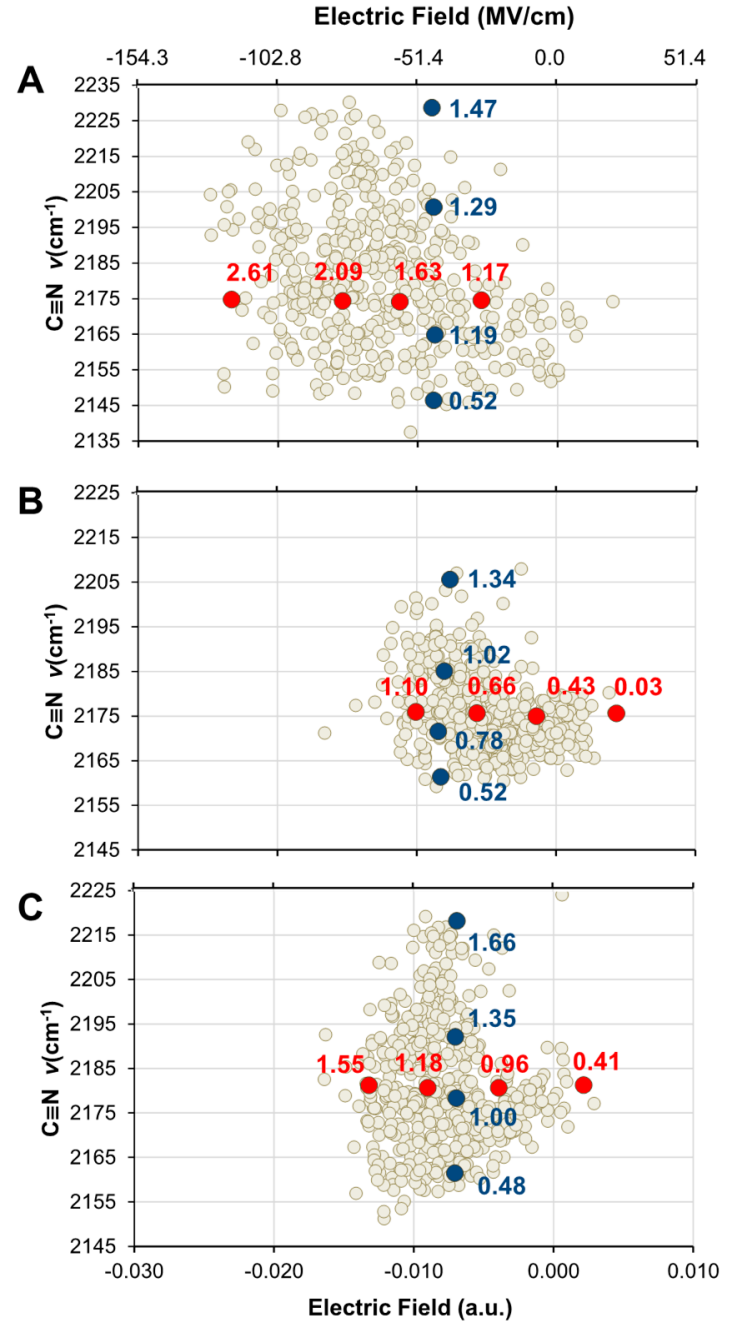

Figure 4. Values of the total electric field projected by EcDHFR on the $\mathrm{C}-\mathrm{N}$ bond for the (A) holoenzyme (E:NADPH), (B) Michaelis complex $\left(\mathrm{E}: \mathrm{NADP}^{+}\right.$:folate), and $(\mathrm{C})$ product ternary complex $\left(\mathrm{E}: \mathrm{NADP}^{+}: \mathrm{THF}\right)$. Blue dots represent selected structures with different nitrile frequencies but the same electric field, while red dots represent selected structures with different electric fields but with the same nitrile frequency. Values on the dots represent hydrogenbonding contributions to the electric field; 1 au $\equiv 5.14225 \times 103$ $\mathrm{MV} / \mathrm{cm}$ (see SI for details).

addition, our analysis reveals that folate produces a counteracting electric field toward the probe, such that the vector of the electric fields produced at the midpoint of the nitrile bond is attenuated in the Michaelis complex as a consequence of substrate binding (Table 1). A similar observation was measured when THF was used. Hence, counteracting electric fields between the cofactor and the substrate could explain the large electrostatic change during the transition between the holoenzyme and the Michaelis complex. Since the Michaelis complex bears significant resemblance to the reaction-ready configuration, we hypothesize that a conducive electrostatic environment for catalysis within the enzyme is attainable only because of the offsetting of the electrostatic interaction between the cofactor and the substrate.

During the transition of EcDHFR from the closed to the occluded conformation, the high electric field propagated by the cofactor becomes partially excluded from the active site (Figure 5), which likely accounts for the overall reduced 
Table 1. QM/MM Theoretical Calculation of the Frequency of the_Nitrile Bond $(\nu)$, Total Electric Field, in au, Projected on the Nitrile Bond $\left(\vec{F}_{\text {all }}\right)$, and Its Contributions from the Cofactor $\left(\vec{F}_{\mathrm{NADP}}\right)$ and the Substrate $\left(\vec{F}_{\text {fol/THF }}\right)^{a}$

EcDHFR complex

E:NADPH

$\mathrm{E}: \mathrm{NADP}^{+}$:folate

$\mathrm{E}: \mathrm{NADP}^{+}: \mathrm{THF}$

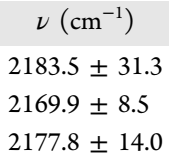

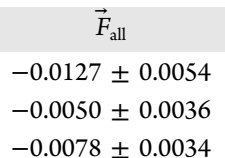

$-0.0050 \pm 0.0036$
$-0.0078 \pm 0.0034$

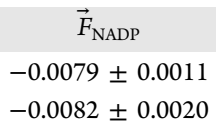

$-0.0069 \pm 0.0009$

\section{$\vec{F}_{\text {fol/THF }}$}

$0.0003 \pm 0.0014$

$0.0017 \pm 0.0009$

${ }^{a}$ Values averaged over 501 structures selected from the $5 \mathrm{~ns}$ classical MD simulations of EcDHFR T46C-CN.

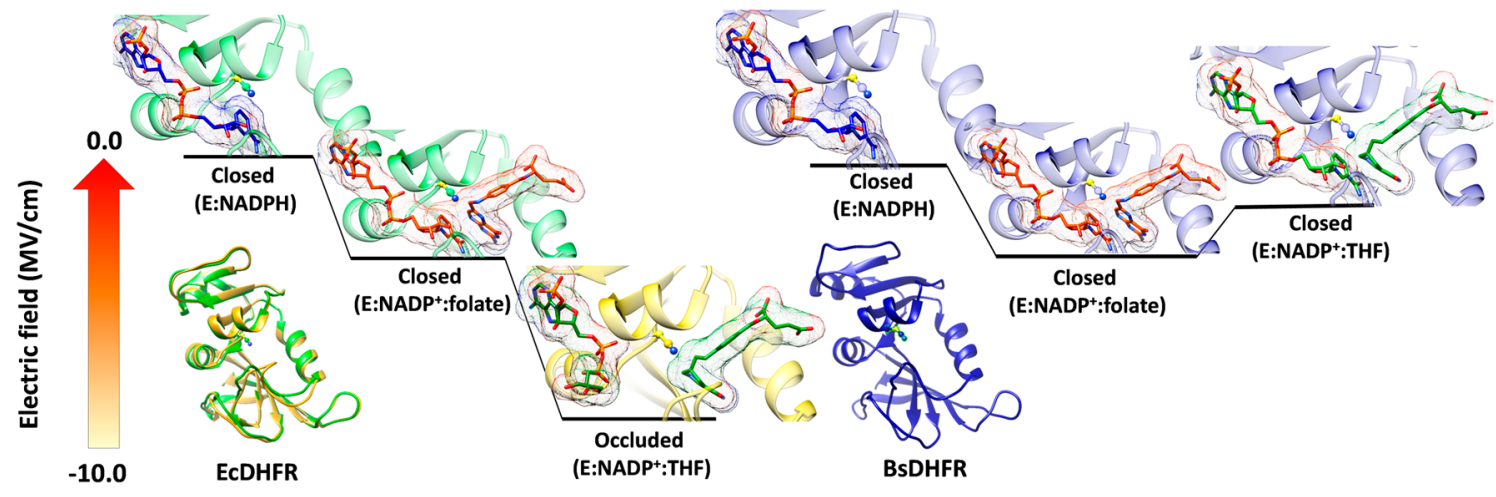

Figure 5. Electric field projected toward the probe in the holoenzyme (E:NADPH), Michaelis complex (E:NADP ${ }^{+}$:folate), and product ternary complex (E:NADP ${ }^{+}$THF) by EcDHFR (left, PDB 4P66, green; PDB 1RX6, gold) and BsDHFR (right; PDB 1ZDR, blue). ${ }^{20}$ Probe was modeled into each structure using the UCSF Chimera software ${ }^{30}$ based on an earlier structure of EcDHFR (PDB 4P66). ${ }^{11}$ Vibrational frequencies of the holoenzymes used as a benchmark for other complexes to calculate the electric field projected toward the probe according to eq 1.

electric field detected by the probe in the occluded conformer. Computational simulation supports this finding and shows that the electric field propagated by the cofactor toward the probe is reduced in the product ternary complex (Table 1). A previous study has suggested that unfavorable ligand-ligand interaction that results from steric clashes between THF and $\mathrm{NADP}^{+}$induces reorganization within the active site of EcDHFR, which leads to exclusion of the cofactor and the protrusion of the M20 loop into the active site. ${ }^{10}$ Unlike EcDHFR, both BsDHFR and EcDHFR-S148P retain the closed conformation in their product ternary complexes because they are not able to form hydrogen bonding between the M20 and the GH loops. ${ }^{20,27}$ This suggests that the ligandligand interaction is not significantly altered between the Michaelis and the product ternary complexes and provides a rationalization for the minor electrostatic changes observed between these complexes in the two enzymes (Figure 5). Product release in BsDHFR is likely mediated by the increased flexibility within its cofactor binding domain, ${ }^{31}$ bypassing the need for significant conformational or electrostatic changes, whereas EcDHFR-S148P exhibits product inhibition. ${ }^{27}$ Hence, our result indicates that modulation of the ligand-ligand electrostatic interaction may, in fact, be a key factor in enzymecatalyzed reactions.

To provide insight into the molecular changes during the chemical step, that is, the transfer of negative charge from the cofactor to the substrate, the electrostatic potential generated in the vicinity of the donor carbon of the cofactor $\left(\mathrm{C}_{4}\right)$ in both the holoenzyme and the Michaelis complex was investigated. The computed values were $-220.6 \pm 15.9$ and $-202.1 \pm 11.0$ $\mathrm{MV} / \mathrm{cm} / \mathrm{e}$ in the holoenzyme and Michaelis complex, respectively. This suggests that the negative charge on $\mathrm{C}_{4}$ of the cofactor has a larger electrostatic stabilization in the holoenzyme than in the Michaelis complex, where an additional stabilizing electrostatic potential $(-173.9 \pm 12.9$ $\mathrm{MV} / \mathrm{cm} / \mathrm{e})$ on the acceptor carbon $\left(\mathrm{C}_{6}\right)$ of the substrate was observed. The electric field projected along the donoracceptor axis generated by the protein, cofactor, and substrate in the Michaelis complex $\left(\mathrm{E}: \mathrm{NADP}^{+}\right.$:folate $)$at the midpoint of the donor and acceptor atoms was calculated and found to be $-13.9 \pm 6.0 \mathrm{MV} / \mathrm{cm}$. In alignment with observations made in a previous study, ${ }^{11}$ the negative vector calculated here indicates that an electrostatic force is applied on the negatively charged $\mathrm{C}_{4}$ of the cofactor in the direction of the substrate, thereby facilitating transfer of the hydride ion. These findings reveal the role of cofactor-substrate electrostatic interaction in modulating electric fields at the midpoint of the reaction center in the holoenzymes and Michaelis and product ternary complexes of DHFRs. Although BsDHFR is less flexible when compared to EcDHFR, it also undergoes substantial electrostatic changes upon substrate binding in the Michaelis complex, where the ligands are brought into close contact. This suggests that ligand-ligand interactions that change along the catalytic cycle drive both the dynamic and the electrostatic changes required for catalysis. This contradicts previous suggestions that the electrostatic properties of DHFRs are mainly controlled by conformational changes during catalysis. ${ }^{4,11-13}$

A previous study has also shown that the orientation and proximity of the cofactor and substrate influence the $\mathrm{p} K_{\mathrm{a}}$ of the substrate when bound in the Michaelis complex. ${ }^{32}$ The binding of the cofactor to the substrate-only complex causes a $\mathrm{p} K_{\mathrm{a}}$ change of ca. 2.5 compared to when only the substrate is bound. ${ }^{33}$ Recently, a theoretical calculation on the beta-kinetic isotope effects on DHFR catalysis reveals that the cofactor polarizes the acceptor atom, increasing its bond length as the net electron density decreases. ${ }^{34}$ Indeed, our simulations predict that adequate orientation of the electrostatic field propagated toward the donor-acceptor axis would favor the transfer of the negatively charged hydride ion. Furthermore, ligand-ligand electrostatic interaction of the cofactor with methotrexate, a major inhibitor of DHFRs, has been reported to enhance the binding of the drug by more than 100 -fold. ${ }^{35}$ 
This indicates that the cofactor-inhibitor electrostatic interaction enhances the drug's effectiveness. Hence, new enzyme inhibitors may be designed that exploit such ligandligand electrostatic interaction to increase potency.

\section{CONCLUSION}

Here, we analyzed the electrostatic changes within the active site of EcDHFR, EcDHFR-S148P, and BsDHFR using VSE and $\mathrm{QM} / \mathrm{MM}$ simulations. A nitrile probe inserted via cysteine post-translational modification to replace an active site residue (Thr46) was found to experience a strong electric field in the enzymes when bound to the cofactor $(\mathrm{NADP}(\mathrm{H}))$. However, this electric field decreases significantly when both the substrate and the cofactor are bound in the Michaelis complex. Computational simulations reveal that the cofactor contributes the major electric field toward the reaction center and that the substrate/product electric field counteracts those of the cofactor. Such a cofactor-substrate interaction is therefore partly absent in the product ternary complex of EcDHFR (Figure 5), accounting for the large electrostatic change between the closed and the occluded conformation. By comparing electric fields within the active site of EcDHFR, EcDHFR-S148P, and BsDHFR, this study contradicts a previous study that suggests the conformational change is relevant to the chemical step of DHFRs. ${ }^{411}$ Instead, the electrostatically preorganized active site contributed by the cofactor-substrate interaction aids the formation of a conducive electrostatic environment for catalysis. While the role of the ligand-ligand interaction may have been observed for enzyme-catalyzed reactions between two identical ligands such as adenylate kinase, ${ }^{36}$ previous work on DHFR and other enzyme models has largely ignored the role of ligand-ligand interactions on catalysis. ${ }^{37,38}$ Provided that most enzymes do not undergo dramatic conformational changes like those that are found in EcDHFR, ${ }^{39}$ the ligand-ligand interaction that controls the electrostatic properties is likely a more crucial factor in the creation of efficient enzymes.

\section{MATERIALS AND METHODS}

Chemicals were purchased from Melford, Apollo Scientific, Sigma, and Fisher Scientific except where otherwise stated. DHF was synthesized as reported previously. ${ }^{40}$

Site-Directed Mutagenesis. Cysteine-free EcDHFR (C85A/C152S) and BsDHFR (C73 V) were used to make further mutations similar to previous studies. ${ }^{41}$ The nucleotides changed for mutagenesis are underlined in the following primer sequences, and the variants are generated using

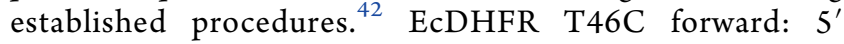
GTGATT ATGGGCCGCCAT TGCTGGGAAT CAATCGGTC 3.' EcDHFR T46C Reverse: 5' GACCGATTGATTCCCA GCAATGGCGGCCCATAATCAC $3^{\prime}$. EcDHFR-S148P forward: 5' CAGAAC CCTCACAGCTATTCTTTTGAGATTCTG GAGC $3{ }^{\prime}$. EcDHFR-S148P reverse: 5' CTGTG AGGGTTCTG CGCATCAGCATCGTG $3^{\prime}$. BsDHFR C73 V forward: 5' GAA GGC GTCCTGGTACTGCATAGCCTGGAAGAAGTG $3^{\prime}$. BsDHFR C73 V reverse 5': GTACCAG GACGCCTTCCGG ACGAAAGCTACGGTTC 3'. BsDHFR T46C forward: 5' CGT AAA TGCTTTGAAGCGATCGGGCGTCCGCT $3^{\prime}$. BsDHFR T46C reverse: 5'CGCTTCAAA GCATTTACGACCCAT TACAATGGCATGACC 3'.
Protein Production, Labeling, and Characterization. Proteins were produced in BL21 (DE3) cells grown in LB media with $100 \mu \mathrm{g} / \mathrm{mL}$ ampicillin at $37^{\circ} \mathrm{C}$. Induction with 0.5 mM IPTG was at O.D. $600 \mathrm{~nm}$ of 0.6 , and the cells were further grown at $20{ }^{\circ} \mathrm{C}$ overnight. Cells were harvested by centrifugation and resuspended in lysis buffer $(50 \mathrm{mM}$ potassium phosphate, $\mathrm{pH} 7.4,1 \mathrm{mM}$ EDTA). Purification was by anion exchange and size exclusion chromatography and concentrations determined by UV absorption at $280 \mathrm{~nm}^{27,43}$ Each enzyme containing a single cysteine residue was separately labeled with 2-nitro-5-thiocyanatobenzoic acid (NTCB) and 5,5'-dithiobis(2-nitrobenzoic acid) (DNTB) for infrared and ${ }^{13} \mathrm{C}$ NMR spectroscopies, respectively. ${ }^{21}$ Reaction progress was monitored at $412 \mathrm{~nm}$ due to release of 2-thio 5nitrobenzoate (TNB) anion. The reactions were washed thrice with $12 \mathrm{mM} \mathrm{KCN} / \mathrm{K}^{13} \mathrm{CN}$ with a concentration step after each wash. The labeled enzyme was finally purified through a Sephadex G-25 column pre-equilibrated with $100 \mathrm{mM}$ potassium phosphate, $\mathrm{pH}$ 7.4. Labeling was confirmed by electron spray ionization MS and further characterization with kinetic analysis and circular dichroism spectroscopy (see SI).

FTIR Spectroscopy. The abortive E:NADP ${ }^{+}$:folate complex is an excellent mimic of the Michaelis complex (E:DHF:NADPH). ${ }^{10}$ The samples were purged with nitrogen and measured at $20{ }^{\circ} \mathrm{C}$ with a variable-temperature demountable liquid cell containing two $\mathrm{CaF}_{2}$ windows separated by a $25 \mu \mathrm{m}$ mylar spacer from Specac Ltd. Spectra were measured with a Vertex $70 \mathrm{~V}$ spectrophotometer equipped with a nitrogen-cooled MCT detector composed of 2000 scans at $1 \mathrm{~cm}^{-1}$ spectral resolution. Spectral treatments used include first derivative, second derivative, and Gaussian fit of the background-subtracted spectra using Origin 9.0 from OriginLab Corp. The average of treatments from at least two independent measurements is reported as the absorption frequency and their standard deviation as error (see SI for details).

Nuclear Magnetic Resonance Spectroscopy. Samples were prepared as previously described with $2.0 \mathrm{mM}$ of $\mathrm{S}^{13} \mathrm{CN}$ labeled protein, $10 \mathrm{mM}$ ligands, with $2.5 \mathrm{mM} 3$-(trimethylsilyl)-1-propanesulfonic acid sodium salt as an external standard. ${ }^{11}$ Samples were purged with nitrogen and protected from light. Measurement was on a Bruker Ultrashield $600 \mathrm{MHz}$ spectrometer equipped with Bruker Topspin software version 4.0.2 at $20^{\circ} \mathrm{C}$.

Computational Simulations. Geometry optimization and frequency calculations of the nitrile bond of $\mathrm{MeCN}$ and $\mathrm{MeSCN}$ in the gas phase were carried out with the Gaussian09 package of programs (see SI). ${ }^{44}$ The Michaelis complex $\left(\mathrm{E}: \mathrm{NADP}^{+}\right.$:folate) was prepared from the initial X-ray structure with PDB code $4 \mathrm{P} 66 .{ }^{11}$ This structure contains a nitrile probe $(\mathrm{XCN})$ attached at position 46 as well as the C85A, D37N, and C152S replacements. NADP ${ }^{+}$and MTX (methotrexate) were in the active site. MTX was manually modified to folate $\left(\mathrm{N}_{4}\right.$ mutated to $\mathrm{O}_{4}$ and $\mathrm{CM}$ removed). When preparing the occluded product ternary complex (E:THF:NADP ${ }^{+}$), the initial structure containing the T46C$\mathrm{CN}$ probe was obtained from $\mathrm{PDB} 1 \mathrm{RX} 6^{10}$ and three replacements were introduced, namely, C85A, C152S, and D37N. The active site contains $\mathrm{NADP}^{+}$and DDF $(5,10-$ dideazatetrahydrofolic acid), which was later modified to THF and the missing ring added to $\mathrm{NADP}^{+}$. The protonation states of titratable residues were determined with PROPKA ver. 3.0 $3{ }^{45}$ The structures of the two states were solvated by placing 
them in a $100 \times 80 \times 80 \AA^{3}$ pre-equilibrated box of water molecules. Five nanoseconds of classical MD simulations were run to equilibrate the systems using the AMBER force field, ${ }^{46}$ as implemented in NAMD software. ${ }^{47}$ Parameters for both substrates were computed with Antechamber at the AM1 level, ${ }^{48}$ while those for the cofactor were from previously published data. ${ }^{49}$ The holoenzyme binary complex E:NADPH was prepared from the Michaelis complex, E:NADP ${ }^{+}$:folate, by removing the substrate and running $5 \mathrm{~ns}$ of classical MD simulations for equilibration (see SI for details). In order to carry out the frequency determination of the nitrile bond, a hybrid QM/MM scheme was used, where the probe was described by a BVP86 functional with the standard 6-311+ $+\mathrm{g}(\mathrm{d}, \mathrm{p})$ basis set and the rest of the protein and water molecules with OPLS-AA ${ }^{50}$ and TIP $3 \mathrm{P}^{51}$ classical force fields, respectively (see SI for details).

\section{ASSOCIATED CONTENT}

\section{SI Supporting Information}

The Supporting Information is available free of charge at https://pubs.acs.org/doi/10.1021/acscatal.0c01856.

Details of experiments for site-directed mutagenesis, protein production and purification, kinetic parameters, mass, CD spectra of purified proteins, FTIR data, tandem FTIR-NMR plots, and computational details (PDF)

\section{AUTHOR INFORMATION}

\section{Corresponding Authors}

Vicent Moliner - Departament de Química Fisica i Analitica, Universitat Jaume I, 12071 Castellón, Spain; 이이.org/ 0000-0002-3665-3391; Email: moliner@uji.es

Rudolf K. Allemann - School of Chemistry, Cardiff University,

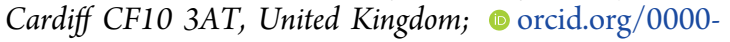
0002-1323-8830; Email: allemannrk@cardiff.ac.uk

\section{Authors}

Aduragbemi S. Adesina - School of Chemistry, Cardiff University, Cardiff CF10 3AT, United Kingdom; (1) orcid.org/ 0000-0002-1029-4159

Katarzyna Świderek - Departament de Química Física i Analitica, Universitat Jaume I, 12071 Castellon, Spain; (1) orcid.org/0000-0002-7528-1551

Louis Y. P. Luk - School of Chemistry, Cardiff University, Cardiff CF10 3AT, United Kingdom; (1) orcid.org/00000002-7864-6261

Complete contact information is available at: https://pubs.acs.org/10.1021/acscatal.0c01856

\section{Author Contributions}

${ }^{\S}$ A.S.A. and K.S.: These authors have contributed equally.

\section{Author Contributions}

The manuscript was written through the contributions of all authors.

\section{Notes}

The authors declare no competing financial interest.

\section{ACKNOWLEDGMENTS}

The authors are grateful to Thomas Williams for technical support and mass spectrometry. K.Ś. acknowledges MINECO for a Juan de la Cierva-Incorporación contract (ref IJCI-201627503). We acknowledge computational resources from the
Servei d'Informàtica of Universitat Jaume I. This work was supported by Cardiff University through a Ph.D. studentship to ASA, the Biotechnology and Biological Sciences Research Council (BBSRC) through grants BB/J005266 and BB/ L020394 (RKA), and the Spanish Ministerio de Ciencia, Innovación y Universidades (Grant PGC2018-094852-B-C21), Generalitat Valenciana (AICO/2019/195), and Universitat Jaume I (project UJıB2017-31).

\section{REFERENCES}

(1) Nakamura, H. Roles of Electrostatic Interaction in Proteins. Q. Rev. Biophys. 1996, 29, 1-90.

(2) Zhou, H. X.; Pang, X. Electrostatic Interactions in Protein Structure, Folding, Binding, and Condensation. Chem. Rev. 2018, 118, $1691-1741$

(3) Head-Gordon, T.; Brooks, C. L. The Role of Electrostatics in the Binding of Small Ligands to Enzymes. J. Phys. Chem. 1987, 91, 33423349.

(4) Hanoian, P.; Liu, C. T.; Hammes-Schiffer, S.; Benkovic, S. Perspectives on Electrostatics and Conformational Motions in Enzyme Catalysis. Acc. Chem. Res. 2015, 48, 482-489.

(5) Schnell, J. R.; Dyson, H. J.; Wright, P. E. Structure, Dynamics, and Catalytic Function of Dihydrofolate Reductase. Annu. Rev. Biophys. Biomol. Struct. 2004, 33, 119-140.

(6) Carey, P. R. Spectroscopic Characterization of Distortion in Enzyme Complexes. Chem. Rev. 2006, 106, 3043-3054.

(7) Belasco, J. G.; Knowles, J. R. Direct Observation of Substrate Distortion by Triosephosphate Isomerase Using Fourier Transform Infrared Spectroscopy. Biochemistry 1980, 19, 472-477.

(8) Luk, L. Y. P.; Loveridge, E. J.; Allemann, R. K. Protein Motions and Dynamic Effects in Enzyme Catalysis. Phys. Chem. Chem. Phys. 2015, 17, 30817-30827.

(9) Bhabha, G.; Ekiert, D. C.; Jennewein, M.; Zmasek, C. M.; Tuttle, L. M.; Kroon, G.; Dyson, H. J.; Godzik, A.; Wilson, I. A.; Wright, P. E. Divergent Evolution of Protein Conformational Dynamics in Dihydrofolate Reductase. Nat. Struct. Mol. Biol. 2013, 20, 1243-1249.

(10) Sawaya, M. R.; Kraut, J. Loop and Subdomain Movements in the Mechanism of Escherichia Coli Dihydrofolate Reductase: Crystallographic Evidence. Biochemistry 1997, 36, 586-603.

(11) Liu, C. T.; Layfield, J. P.; Stewart, R. J.; French, J. B.; Hanoian, P.; Asbury, J. B.; Hammes-Schiffer, S.; Benkovic, S. J. Probing the Electrostatics of Active Site Microenvironments along the Catalytic Cycle for Escherichia Coli Dihydrofolate Reductase. J. Am. Chem. Soc. 2014, 136, 10349-10360.

(12) Groff, D.; Thielges, M. C.; Cellitti, S.; Schultz, P. G.; Romesberg, F. E. Efforts toward the Direct Experimental Characterization of Enzyme Microenvironments: Tyrosine100 in Dihydrofolate Reductase. Angew. Chem., Int. Ed. 2009, 48, 3478-3481.

(13) Thielges, M.; Case, D. A.; Romesberg, F. E. Carbon- Deuterium Bonds as Probes of Dihydrofolate Reductase. J. Am. Chem. Soc. 2008, 130, 6597-6603.

(14) Hay, S.; Evans, R. M.; Levy, C.; Loveridge, E. J.; Wang, X.; Leys, D.; Allemann, R. K.; Scrutton, N. S. Are the Catalytic Properties of Enzymes from Piezophilic Organisms Pressure Adapted? ChemBioChem 2009, 10, 2348-2353.

(15) Mhashal, A. R.; Vardi-Kilshtain, A.; Kohen, A.; Major, D. T. The Role of the Met 20 Loop in the Hydride Transfer in Escherichia Coli Dihydrofolate Reductase. J. Biol. Chem. 2017, 292, 1422914239.

(16) Mhashal, A. R.; Pshetitsky, Y.; Cheatum, C. M.; Kohen, A.; Major, D. T. Evolutionary Effects on Bound Substrate PKa in Dihydrofolate Reductase. J. Am. Chem. Soc. 2018, 140, 16650-16660.

(17) Dams, T.; Jaenicke, R. Dihydrofolate Reductase from Thermotoga Maritima. Methods Enzymol. 2001, 331, 305-317.

(18) Fried, S. D.; Boxer, S. G. Electric Fields and Enzyme Catalysis. Annu. Rev. Biochem. 2017, 86, 387-415.

(19) Deb, P.; Haldar, T.; Kashid, S. M.; Banerjee, S.; Chakrabarty, S.; Bagchi, S. Correlating Nitrile IR Frequencies to Local Electro- 
statics Quantifies Non-Covalent Interactions of Peptides and Proteins.

J. Phys. Chem. B 2016, 120, 4034-4046.

(20) Kim, H. S.; Damo, S. M.; Lee, S. Y.; Wemmer, D.; Klinman, J. P. Structure and Hydride Transfer Mechanism of a Moderate Thermophilic Dihydrofolate Reductase from Bacillus Stearothermophilus and Comparison to Its Mesophilic and Hyperthermophilic Homologues. Biochemistry 2005, 44, 11428-11439.

(21) Fafarman, A. T.; Webb, L. J.; Chuang, J. I.; Boxer, S. G. Site Specific Conversion of Cysteine Thiols into Thiocyanate Creates an IR Probe for Electric Fields in Proteins. J. Am. Chem. Soc. 2006, 128, 13356-13357.

(22) Webb, L. J.; Boxer, S. G. Electrostatic Fields Near the Active Site of Human Aldose Reductase: 1. New Inhibitors and Vibrational Stark Effect Measurements. Biochemistry 2008, 47, 1588-1598.

(23) Fafarman, A. T.; Sigala, P. A.; Schwans, J. P.; Fenn, T. D.; Herschlag, D.; Boxer, S. G. Quantitative, Directional Measurement of Electric Field Heterogeneity in the Active Site of Ketosteroid Isomerase. Proc. Natl. Acad. Sci. U. S. A. 2012, 109, E299-E308.

(24) Fried, S. D.; Boxer, S. G. Measuring Electric Fields and Noncovalent Interactions Using the Vibrational Stark Effect. Acc. Chem. Res. 2015, 48, 998-1006.

(25) Fafarman, A. T.; Sigala, P. A.; Herschlag, D.; Boxer, S. G. Decomposition of Vibrational Shifts of Nitriles into Electrostatic and Hydrogen-Bonding Effects. J. Am. Chem. Soc. 2010, 132, 1281112813.

(26) Falzone, C. J.; Benkovic, S. J.; Wright, P. E. Dynamics of a Flexible Loop in Dihydrofolate Reductase from Escherichia Coli and Its Implication for Catalysis. Biochemistry 1994, 33, 439-442.

(27) Behiry, E. M.; Luk, L. Y. P.; Matthews, S. M.; Loveridge, E. J.; Allemann, R. K. Role of the Occluded Conformation in Bacterial Dihydrofolate Reductases. Biochemistry 2014, 53, 4761-4768.

(28) Layfield, J. P.; Hammes-Schiffer, S. Calculation of Vibrational Shifts of Nitrile Probes in the Active Site of Ketosteroid Isomerase upon Ligand Binding. J. Am. Chem. Soc. 2013, 135, 717-725.

(29) Pu, J.; Gao, J.; Truhlar, D. G. Multidimensional Tunneling, Recrossing, and the Transmission Coefficient for Enzymatic Reactions. Chem. Rev. 2006, 106, 3140-3169.

(30) Pettersen, E. F.; Goddard, T. D.; Huang, C. C.; Couch, G. S.; Greenblatt, D. M.; Meng, E. C.; Ferrin, T. E. UCSF Chimera: A Visualization System for Exploratory Research and Analysis. J. Comput. Chem. 2004, 25, 1605-1612.

(31) Oyeyemi, O. A.; Sours, K. M.; Lee, T.; Kohen, A.; Resing, K. A.; Ahn, N. G.; Klinman, J. P. Comparative Hydrogen-Deuterium Exchange for a Mesophilic vs Thermophilic Dihydrofolate Reductase at $25{ }^{\circ} \mathrm{C}$ : Identification of a Single Active Site Region with Enhanced Flexibility in the Mesophilic Protein. Biochemistry 2011, 50, 82518260.

(32) Khavrutskii, I. V.; Price, D. J.; Lee, J.; Brooks, C. L. Conformational Change of the Methionine 20 Loop of Escherichia Coli Dihydrofolate Reductase Modulates PKa of the Bound Dihydrofolate. Protein Sci. 2007, 16, 1087-1100.

(33) Chen, Y. Q.; Kraut, J.; Callender, R. PH-Dependent Conformational Changes in Escherichia Coli Dihydrofolate Reductase Revealed by Raman Difference Spectroscopy. Biophys. J. 1997, 72, 936-941.

(34) Angelastro, A.; Ruiz-Pernía, J. J.; Tuñón, I.; Moliner, V.; Luk, L. Y. P.; Allemann, R. K. Loss of Hyperconjugative Effects Drives Hydride Transfer during Dihydrofolate Reductase Catalysis. ACS Catal. 2019, 9, 10343-10349.

(35) Kamen, B. A.; Whyte-Bauer, W.; Bertino, J. R. A Mechanism of Resistance to Methotrexate NADPH but Not NADH Stimulation of Methotrexate Binding to Dihydrofolate Reductase. Biochem. Pharmacol. 1983, 32, 1837-1841.

(36) Morris, H. P.; Criss, W. E. Kinetic Regulation of Adenylate Kinases from Muscle, Liver, and Hepatoma. Cancer Res. 1974, 34, 3058-3061.

(37) Garcia-Viloca, M.; Truhlar, D. G.; Gao, J. Importance of Substrate and Cofactor Polarization in the Active Site of Dihydrofolate Reductase. J. Mol. Biol. 2003, 327, 549-560.
(38) Bajorath, J.; Kitson, D. H.; Fitzgerald, G.; Andzelm, J.; Kraut, J.; Hagler, A. T. Electron Redistribution on Binding of a Substrate to an Enzyme: Folate and Dihydrofolate Reductase. Proteins: Struct., Funct., Genet. 1991, 9, 217-224.

(39) Gutteridge, A.; Thornton, J. Conformational Change in Substrate Binding, Catalysis and Product Release: An Open and Shut Case? FEBS Lett. 2004, 567, 67-73.

(40) Maglia, G.; Allemann, R. K. Evidence for Environmentally Coupled Hydrogen Tunneling during Dihydrofolate Reductase Catalysis. J. Am. Chem. Soc. 2003, 125, 13372-13373.

(41) Swanwick, R. S.; Daines, A. M.; Tey, L.-H.; Flitsch, S. L.; Allemann, R. K. Increased Thermal Stability of Site-Selectively Glycosylated Dihydrofolate Reductase. ChemBioChem 2005, 6, 13381340.

(42) Liu, H.; Naismith, J. H. An Efficient One-Step Site-Directed Deletion, Insertion, Single and Multiple-Site Plasmid Mutagenesis Protocol. BMC Biotechnol. 2008, 8, 91-99.

(43) Luk, L. Y. P.; Ruiz-Pernía, J. J.; Dawson, W. M.; Loveridge, E. J.; Tuñón, I.; Moliner, V.; Allemann, R. K. Protein Isotope Effects in Dihydrofolate Reductase from Geobacillus Stearothermophilus Show Entropic-Enthalpic Compensatory Effects on the Rate Constant. J. Am. Chem. Soc. 2014, 136, 17317-17323.

(44) Frisch, M. J.; Trucks, G. W.; Schlegel, H. B.; Scuseria, G. E.; Robb, M. A.; Cheeseman, J. R.; Scalmani, G.; Barone, V.; Mennucci, B.; Petersson, G. A.; Nakatsuji, H.; Caricato, M.; Li, X.; Hratchian, H. P.; Izmaylov, A. F.; Bloino, J.; Zheng, G.; Sonnenberg, J. L.; Hada, M.; Ehara, M.; Toyota, K.; Fukuda, R.; Hasegawa, J.; Ishida, M.; Nakajima, T.; Honda, Y.; Kitao, O.; Nakai, H.; Vreven, T.; Montgomery, J. A. J.; Peralta, J. E.; Ogliaro, F.; Bearpark, M.; Heyd, J. J.; Brothers, E.; Kudin, K. N.; Staroverov, V. N.; Kobayashi, R.; Normand, J.; Raghavachari, K.; Rendell, A.; Burant, J. C.; Iyengar, S. S.; Tomasi, J.; Cossi, M.; Rega, N.; Millam, N. J.; Klene, M.; Knox, J. E.; Cross, J. B.; Bakken, V.; Adamo, C.; Jaramillo, J.; Gomperts, R.; Stratmann, R. E.; Yazyev, O.; Austin, A. J.; Cammi, R.; Pomelli, C.; Ochterski, J. W.; Martin, R. L.; Morokuma, K.; Zakrzewski, V. G.; Voth, G. A.; Salvador, P.; Dannenberg, J. J.; Dapprich, S.; Daniels, A. D.; Farkas, Ö.; Foresman, J. B.; Ortiz, J. V.; Cioslowski, J.; Fox, D. J. Gaussian 09; Gaussian, Inc.: Wallingford, CT, 2009.

(45) Olsson, M. H. M.; Søndergaard, C. R.; Rostkowski, M.; Jensen, J. H. PROPKA3: Consistent Treatment of Internal and Surface Residues in Empirical PK a Predictions. J. Chem. Theory Comput. 2011, 7, 525-537.

(46) Duan, Y.; Wu, C.; Chowdhury, S.; Lee, M. C.; Xiong, G.; Zhang, W.; Yang, R.; Cieplak, P.; Luo, R.; Lee, T.; Caldwell, J.; Wang, J.; Kollman, P. A Point-Charge Force Field for Molecular Mechanics Simulations of Proteins Based on Condensed-Phase. J. Comput. Chem. 2003, 24, 1999-2012.

(47) Phillips, J. C.; Braun, R.; Wang, W.; Gumbart, J.; Tajkhorshid, E.; Villa, E.; Chipot, C.; Skeel, R. D.; Kale, L.; Schulten, K. Scalable Molecular Dynamics with NAMD. J. Comput. Chem. 2005, 26, 17811802.

(48) Wang, J.; Wang, W.; Kollman, P. A.; Case, D. A. Automatic Atom Type and Bond Type Perception in Molecular Mechanical Calculations. J. Mol. Graphics Modell. 2006, 25, 247-260.

(49) Cummins, P. L.; Ramnarayan, K.; Gready, J. E.; Singh, U. C. Molecular Dynamics/Free Energy Perturbation Study on the Relative Affinities of the Binding of Reduced and Oxidized NADP to Dihydrofolate Reductase. J. Am. Chem. Soc. 1991, 113, 8247-8256.

(50) Jorgensen, W. L.; Maxwell, D. S.; Tirado-Rives, J. Development and Testing of the OPLS All-Atom Force Field on Conformational Energetics and Properties of Organic Liquids. J. Am. Chem. Soc. 1996, $118,11225-11236$.

(51) Jorgensen, W. L.; Chandrasekhar, J.; Madura, J. D.; Impey, R. W.; Klein, M. L. Comparison of Simple Potential Functions for Simulating Liquid Water. J. Chem. Phys. 1983, 79, 926-935. 